\title{
Transistor Noise in SiGe HBT RF Technology
}

\author{
Guofu Niu, Zhenrong Jin, John D. Cressler, Rao Rapeta, Alvin J. Joseph, and David Harame
}

\begin{abstract}
This brief presents experimental and modeling results of device noise in SiGe HBT RF technology. By careful bandgap engineering, a simultaneous reduction of $\mathrm{RF}$ noise, $1 / f$ noise, and phase noise has been achieved. At a given $I_{B}$, transistors with different base bandgap profiles show similar $1 / f$ noise. At a given $I_{C}$, however, transistors with a higher $\beta$ (and hence lower RF noise) show lower $1 / f$ noise. Circuit analysis and simulation shows that the phase noise is reduced as well.
\end{abstract}

Index Terms-Bipolar technology, flicker noise, modeling, phase noise, RF circuits, RF noise, SiGe HBT.

\section{INTRODUCTION}

$\mathbf{M}$ OBILE wireless communication links demand very sensitive and highly selective transceiver circuits. Key performance metrics for wireless RF links include: dynamic range, sensitivity, and selectivity. Dynamic range and sensitivity heavily depend on the RF noise and linearity of the transistors used in the low-noise amplifiers (LNAs). Small improvements of noise at the device level can have a large impact on overall system performance. For instance, a degradation of LNA noise figure by even $0.5 \mathrm{~dB}$ can be very detrimental to the RF link performance. On the other hand, selectivity depends on the local oscillator (LO) phase noise, which in turn depends on the phase noise capability of the transistors used, and to a large extent is determined by the low-frequency noise (also known as flicker noise or $1 / f$ noise). Direct-conversion transceivers also demand transistors with extremely low $1 / f$ noise [1].

It was shown earlier that the RF noise in SiGe HBTs can be reduced by careful optimization of SiGe profile [2], [3]. In this work, we examine the impact of such SiGe profile optimization on $1 / f$ noise and phase noise capability of these SiGe HBTs. Details of the SiGe HBT technology can be found in [4].

\section{RF NOISE}

The primary RF noise sources in a bipolar transistor are the base current shot noise $2 q I_{B}$, the collector current shot noise $2 q I_{C}$, and the base resistance-induced thermal noise $4 k T r_{B} \cdot \mathrm{A}$ higher base doping and a smaller emitter width are thus desired to reduce $r_{B}$, which implies the need to move to a more aggressive technology with a lower thermal cycle. The net contribution

Manuscript received December 12, 2000; revised March 20, 2001. This work was supported by IBM under a University Partnership Research Award, the Alabama Microelectronics Science and Technology Center, and the Semiconductor Research Corporation under Contract SRC 2000-HJ-769.

G. Niu, Z. Jin, J. D. Cressler, and R. Rapeta are with the Alabama Microelectronics Science and Technology Center, Electrical and Computer Engineering Department, Auburn University, Auburn, AL 36849 USA (e-mail: guofu@eng.auburn.edu).

A. J. Joseph and D. Harame are with IBM Microelectronics, Essex Junction, VT 05401 USA.

Publisher Item Identifier S 0018-9200(01)06109-1.
TABLE I

SUMMARY OF DEVICE ElECTRICAL CHARACTERISTICS

\begin{tabular}{l||c|c|c|c}
\hline Performance & $\begin{array}{c}\text { Si } \\
\text { BJT }\end{array}$ & $\begin{array}{c}\text { SiGe } \\
\text { control }\end{array}$ & $\begin{array}{c}\text { SiGe } \\
\text { LN1 }\end{array}$ & $\begin{array}{c}\text { SiGe } \\
\text { LN2 }\end{array}$ \\
\hline \hline$\beta$ at $V_{B E}=0.7 \mathrm{~V}$ & 67 & 114 & 350 & 261 \\
\hline$V_{A}(\mathrm{~V})$ & 19 & 60 & 58 & 113 \\
\hline$B V_{C E O}(V)$ & 3.5 & 3.2 & 2.7 & 2.7 \\
\hline$R_{B i}(k \Omega / \square)$ & 12.8 & 9.8 & 10.3 & 10.7 \\
\hline peak $f_{T}(\mathrm{GHz})$ & 38 & 52 & 52 & 57 \\
\hline peak $f_{\max }(\mathrm{GHz})$ & 57 & 64 & 62 & 67 \\
\hline
\end{tabular}

of the base and collector current shot noise, however, can be reduced by increasing $\beta$ (to reduce $I_{B}$ ) and increasing $f_{T}$ (to increase $h_{21}$ ), both of which can be realized by SiGe profile optimization at a given technology generation [2], [3]. The resulting bandgap profiles in the base increase the current gain and speed. In particular, $2 q I_{B}$ dominates the effective input noise current at relatively high $I_{C}$ where RF gain is large. Noise improvement can only be achieved through the increase of $\beta$ at those relatively high currents. The superior RF noise performance of HBTs lies in their ability to simultaneously achieve high $\beta$, high $f_{T}$, and low $r_{B}$.

We consider in the following four types of transistors: a control SiGe HBT, two low-noise SiGe HBTs (LN1 and LN2) with optimized SiGe profiles, and an Si BJT for comparison. Details of the SiGe profile optimization and related simulation issues can be found in [2]-[5]. All of the transistors were fabricated in the same wafer lot under identical processing conditions. Compared to the SiGe control HBT, LN1 and LN2 have a higher Ge content and a larger Ge gradient in the neutral base, but less $\mathrm{Ge}$ retrograding into the collector in order to keep the total amount of Ge within the SiGe film thermal stability limit. LN1 and LN2 thus have a much higher $\beta$, a slightly higher $f_{T}$, and hence lower $N F_{\text {min }}$ at the expense of a stronger $f_{T}$ roll-off at very high currents [2]. The peak Ge mole fraction is $10 \%, 14 \%$, and $18 \%$ in the SiGe control, LN1, and LN2 HBTs, respectively. A summary of device electrical characteristics is given in Table I. The LN1 and LN2 HBTs achieve an impressive $N F_{\text {min }}$ of $0.2 \mathrm{~dB}$ at $2 \mathrm{~mA}, 0.2 \mathrm{~dB}$ lower than the SiGe control profile [3].

\section{LOW-FreQUENCY NOISE AND PHASE NoISE}

SiGe HBTs have not only low RF noise, but also low phase noise [6]. It is logical to wonder how the SiGe profile optimization for RF noise improvement affects transistor phase noise capability. To answer this question, we need to examine 


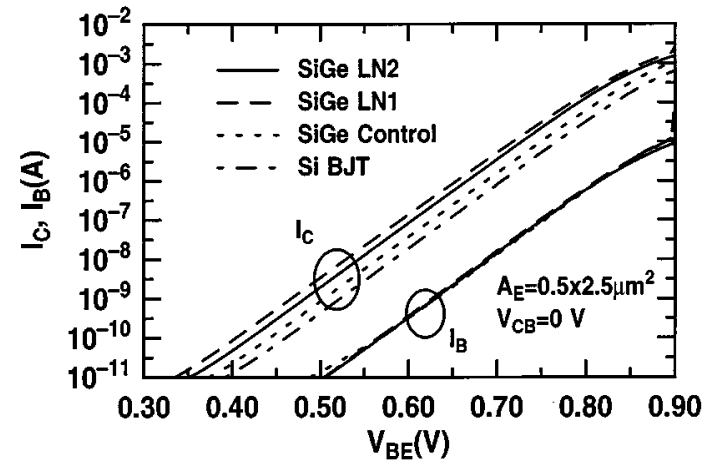

Fig. 1. Measured $I_{C}$ and $I_{B}$ versus $V_{B E}$ for the Si BJT, SiGe control, and two low-noise SiGe HBTs.

the impact of bandgap engineering on low-frequency noise and device linearity (distortion), because physically phase noise is upconverted from low-frequency noise through device nonlinearities. In a transistor amplifier, the low-frequency noise is mixed with the RF signal through the physical nonlinearities (e.g., $I_{C}-V_{B E}$ nonlinearity), which in turn leads to residual amplitude and phase noise at the output. The residual phase noise is translated into oscillator phase noise by the loop effect [7]. The amplitude noise is generally not important for oscillators because of the inherent stabilization. Load-pull measurements showed that the four types of transistors investigated in this work with various base bandgap profiles have nearly identical linearity [2], [3]. We will therefore focus on the impact of bandgap engineering on $1 / f$ noise.

It has been established experimentally that only the base current has $1 / f$ noise in bipolar transistors. The noise is typically proportional to $I_{B}^{\alpha}$ and inversely proportional to the emitter junction area $A_{e}$

$$
S_{I_{B}}=\frac{K}{A_{e}} I_{B}^{\alpha} \frac{1}{f}
$$

where $K$ is a technology-dependent constant, and $\alpha$ has a typical value of 2 in modern transistors. The physical origin of $1 / f$ noise is the mobility or number fluctuation (or both) of the carriers due to defects. Assuming that the $1 / f$ noise process is solely determined by the number of minority carriers injected into the emitter, we intuitively expect the same $1 / f$ noise at a given $V_{B E}$ (and hence a given $I_{B}$ ) in transistors with different base bandgap profiles. At the same $V_{B E}$, a SiGe HBT has a higher $I_{C}$ than its Si BJT counterpart because of a narrower base bandgap. However, a SiGe HBT has the same $I_{B}$ as a Si BJT at a given $V_{B E}$, because of the identical emitter structure. $I_{B}$ is dominated by hole injection into the emitter in modern bipolar transistors. The measured $I_{C}$ and $I_{B}$ as a function of $V_{B E}$ of the four profiles are shown in Fig. 1. At a given $V_{B E}$, the two low-noise SiGe HBTs have higher $I_{C}$, and hence higher $\beta$, as expected.

To confirm the above speculation, extensive low-frequency noise measurements were made on the four kinds of transistors. From a pure device physics point of view, a comparison at constant $V_{B E}\left(I_{B}\right)$ makes better sense, because it provides information on the rate of mobility or number fluctuation. However, from an RF circuit point of view, a comparison at constant $I_{C}$ is

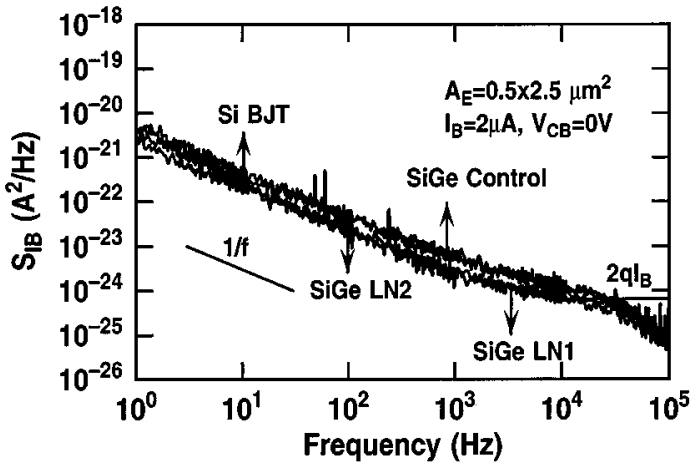

Fig. 2. $S_{I_{B}}$ spectra for the Si BJT, SiGe control, and two low-noise HBTs measured at constant $I_{B}$.

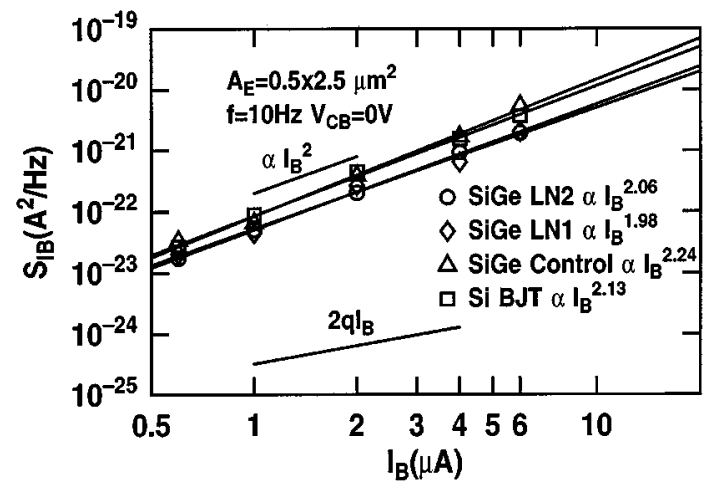

Fig. 3. Measured $S_{I_{B}}$ at $10 \mathrm{~Hz}$ as a function of $I_{B}$ for the Si BJT, SiGe control, and two low-noise HBTs.

more meaningful, because many RF figures-of-merit fundamentally depend on $I_{C}$ instead of $I_{B}$ (e.g., $f_{T}$ and $f_{\max }$ ). $N F_{\min }$, though dependent on $I_{B}$, is often compared at the same $I_{C}$ as well. Devices with an emitter area of $0.5 \times 2.5 \mu \mathrm{m}^{2}$ are used here due to the power supply limitation of the experimental $1 / f$ noise measurement setup.

\section{A. Low-Frequency Noise at Constant $I_{B}$}

Fig. 2 shows the measured base current low-frequency noise spectra $\left(S_{I_{B}}\right)$ at a constant base current of $2 \mu \mathrm{A}$. The noise spectra are all of $1 / f$ type, and are nearly identical at this base current for the four kinds of transistors, despite the difference in base bandgap profile. The measured $S_{I_{B}}$ at $10 \mathrm{~Hz}$ are shown as a function of $I_{B}$ in Fig. 3. With increasing base current, these transistors start to show a larger difference in $1 / f$ noise at constant $I_{B}$. The two low-noise SiGe HBTs show a slightly lower $1 / f$ noise than the SiGe control and the Si BJT at higher $I_{B}$. A typical $I_{B}^{\alpha}$ dependence is observed, with $\alpha$ ranging from 1.98 in LN1 SiGe HBT to 2.24 in the SiGe control.

\section{B. Low-Frequency Noise at Constant $I_{C}$}

As discussed earlier, a constant $I_{C}$ comparison is more meaningful for RF circuit applications. For example, the minimum noise figure is often compared at the same $I_{C}$ for a Si BJT and a SiGe HBT. At the same $I_{C}$ (DC power), the SiGe HBTs can be used for lower RF noise, because of the lower base shot noise $2 q I_{B}$. A benefit of reduced $1 / f$ noise comes naturally with this $I_{B}$ reduction when comparison is made at the same collector 


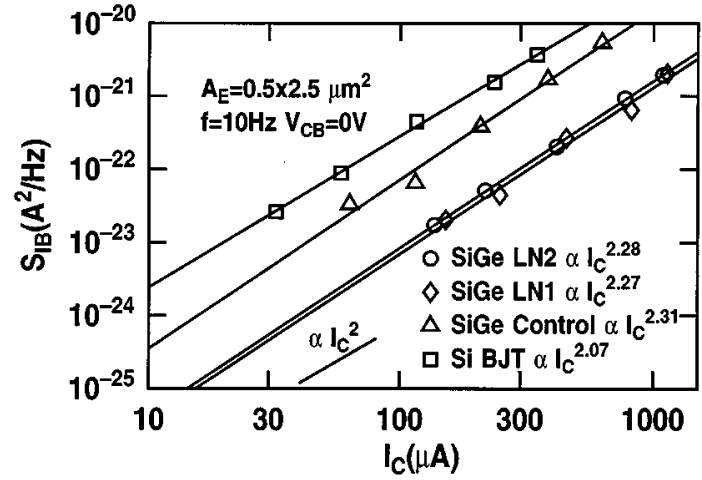

Fig. 4. Measured $S_{I_{B}}$ at $10 \mathrm{~Hz}$ as a function of $I_{C}$ for the Si BJT, SiGe control, and two low-noise HBTs.

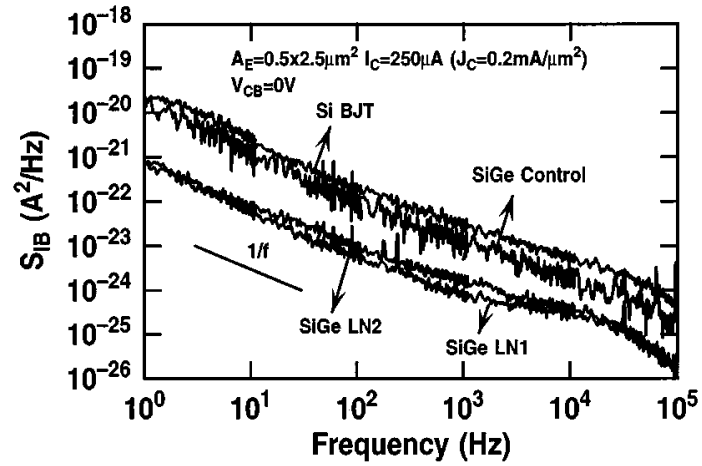

Fig. 5. $S_{I_{B}}$ spectra for the Si BJT, SiGe control, and two low-noise HBTs measured at the same $I_{C}$.

current $\left(I_{C}\right.$ ). The higher $\beta$ corresponds to a smaller $I_{B}$ (or $V_{B E}$ ) because of fixed $I_{C}$, and thus a smaller $S_{I_{B}}$, as can be seen from Fig. 4.

Fig. 5 shows the measured base current low-frequency noise spectra at a constant $I_{C}$ of $0.25 \mathrm{~mA}$, which corresponds to a current density where low-noise amplifiers are typically biased $\left(0.2 \mathrm{~mA} / \mu \mathrm{m}^{2}\right)$ in this technology. As expected, the $1 / f$ noise is much smaller in the two low-noise SiGe HBTs than in the $\mathrm{SiGe}$ control HBT and Si control BJT. Consequently, we expect a lower residual phase noise in those two low-noise $\mathrm{SiGe}$ HBT's, and hence simultaneous improvement in both RF noise and phase noise using bandgap engineering. This is an important result if verified.

\section{Impact on Phase Noise}

To verify the above result, the phase noise of a single-transistor amplifier is simulated using HP-ADS. A calibrated VBIC model was used. The model parameters were extracted from the measured dc data, $s$-parameters, and low-frequency noise data. Higher order effects including self-heating and avalanche multiplication were taken into account in the VBIC model. The source and load were both terminated at $50 \Omega$. Fig. 6 shows the comparison of the simulated phase noise for a $2-\mathrm{GHz}$ carrier at $I_{C}=10 \mathrm{~mA}\left(A_{e}=0.5 \times 20 \times 2 \mu \mathrm{m}^{2}\right)$. The reduced $1 / f$ noise in the LN1 and LN2 HBTs is translated into a reduction of phase noise at the output. The Si BJT has the highest phase noise because of the highest $1 / f$ noise at a given $I_{C}$. The power gains

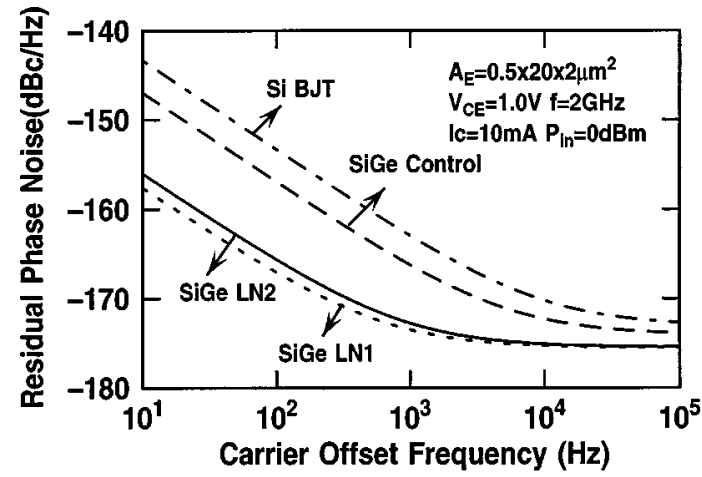

Fig. 6. Simulated phase noise spectra of a single-transistor amplifier for the Si BJT, SiGe control, and two low-noise HBTs at $I_{C}=20 \mathrm{~mA} . A_{\epsilon}=0.5 \times$ $20 \times 2 \mu \mathrm{m}^{2}$. The source and load terminations are both $50 \Omega$. The input power is $0 \mathrm{dBm}$.

in the HBTs (LN1, LN2, and SiGe control) are nearly identical, and higher than for the Si BJT.

To gain intuitive understanding of the simulated results, let us examine the nonlinear equivalent circuit of a bipolar transistor in Fig. 7. To simplify analysis, we neglect the CB capacitance and avalanche multiplication. An examination of the equivalent circuit including those higher order effects will show that they do not change the conclusion, as confirmed by simulation. At a given $I_{C}$, the impact of $I_{B}$ on the nonlinear circuit is twofold:

1) $I_{B}$ determines the magnitude of $1 / f$ noise $S_{I_{B}}$, which is approximately proportional to $I_{B}^{2}$. The incoming RF signal is modulated or mixed by the $1 / f$ noise through nonlinear circuit elements. As a result, phase noise is generated at the output. The high $\beta$ HBTs therefore have a natural advantage of smaller $1 / f$ noise for upconversion into phase noise.

2) $I_{B}$ determines the nonlinear base current $i_{1}$, which can be approximated by a Taylor series [8]

$$
i_{1}=\frac{I_{B}}{V_{t}} v_{b e}+\frac{I_{B}}{2 V_{t}^{2}} v_{b e}^{2}+\frac{I_{B}}{6 V_{t}^{3}} v_{b e}^{3}+\cdots
$$

where $V_{t}=k T / q$ is the thermal voltage. At RF frequencies, the conductance through the nonlinear EB capacitance $C_{b e}\left(i_{2}\right)$ dominates over $i_{1}$. Thus, $i_{1}$, and hence $I_{B}$, has no impact on the upconversion process.

The nonlinear emitter-base capacitive current $i_{2}$ is related to $v_{b e}$ by

$$
i_{2}=\frac{d}{d t}\left\{C_{b e} v_{b e}+K_{2 C_{b e}} v_{b e}^{2}+K_{3 C_{b e}} v_{b e}^{3}+\cdots\right\}
$$

where $K_{2 C_{b e}}$ and $K_{3 C_{b e}}$ are constants determined by the second- and third-order derivative of $C_{b e}$ with respect to $v_{b e}$, respectively [8]. The nonlinear collector current $i_{3}$ is given by

$$
i_{3}=\frac{I_{C}}{V_{t}} v_{b e}+\frac{I_{C}}{2 V_{t}^{2}} v_{b e}^{2}+\frac{I_{C}}{6 V_{t}^{3}} v_{b e}^{3}+\cdots .
$$

At the same $I_{C}, C_{b e}, K_{2 C_{b e}}$ and $K_{3 C_{b e}}$ are approximately the same for the three SiGe HBTs, because of similar transit time. For the same $i_{2}, v_{b e}$ is the same because of negligible $i_{1}$, resulting in the same upconversion gain $\left(i_{3}\right)$. The $\beta$ of LN1 and 


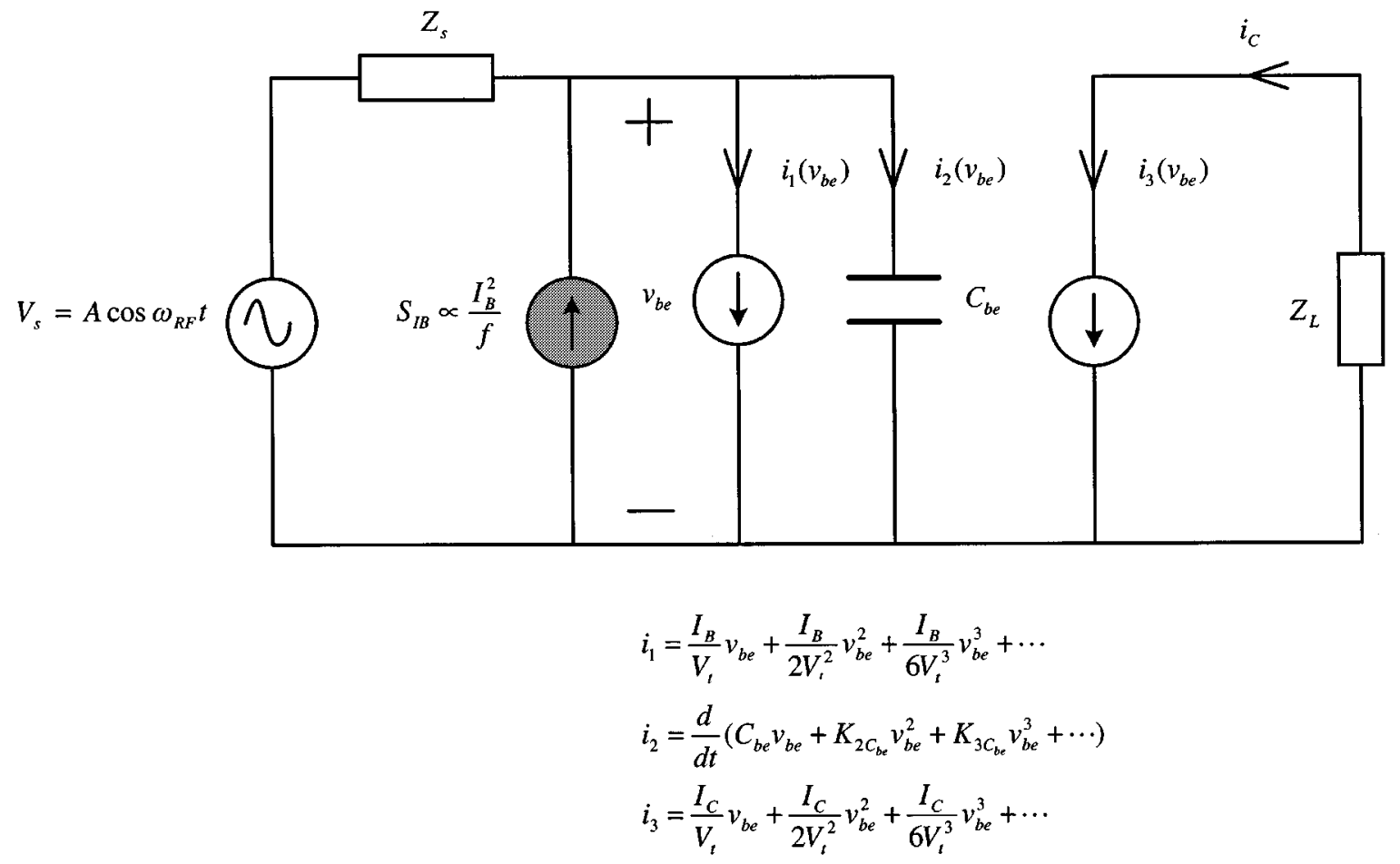

Fig. 7. A simplified nonlinear equivalent circuit of a bipolar transistor.

LN2 HBTs, however, are much higher than that of the SiGe control. Consequently, at a given $I_{C}$, a higher $\beta$ reduces the $1 / f$ noise, the source for upconversion into phase noise (part of $i_{2}$ ). As a result, the phase noise generated at the output is smaller for LN1 and LN2 HB's because of the same upconversion gain.

The bandgap engineering for RF noise improvement is thus beneficial to the phase noise capability of these HBT's, as confirmed by both simulation and circuit analysis. A simultaneous improvement in RF noise and phase noise has been achieved using bandgap engineering. This, we believe, is an important result, and has significant implications on RF circuits demanding both low RF noise and low phase noise.

\section{CONCLUSION}

We have presented experimental and modeling results of device noise in a SiGe HBT RF technology. Bandgap engineering through SiGe profile optimization not only improves the RF noise performance, but also improves the phase noise capability for circuit applications. At a given $I_{B}$, transistors with different base bandgap profiles show similar $1 / f$ noise. At a given $I_{C}$, however, transistors with a higher $\beta$ (and hence lower RF noise) show smaller $1 / f$ noise. This natural reduction of $1 / f$ noise reduces phase noise as well, as shown by simulation.

\section{ACKNOWLEDGMENT}

The wafers were fabricated at IBM Microelectronics, Essex Junction, VT. The authors would like to thank D. Ahlgren, S. Subbanna, B. Meyerson, and D. Herman for their support of this work.

\section{REFERENCES}

[1] A. Abidi, "Low-power radio frequency IC's for portable communications," in RF/Microwave Circuit Design for Wireless Communications, L. Larson, Ed. Norwood, MA: Artech House, 1996, pp. 43-98.

[2] G. F. Niu, S. Zhang, J. D. Cressler, A. J. Joseph, J. S. Fairbanks, L. E. Larson, C. S. Webster, W. E. Ansley, and D. L. Harame, "SiGe profile design tradeoffs for RF circuit applications," in Tech. Dig. IEDM, 1999, pp. $573-576$.

[3] _ "Noise modeling and SiGe profile design tradeoffs for RF applications," IEEE Trans. Electron Devices, vol. 47, pp. 2037-2044, 2000.

[4] S. Subbanna et al., "Integration and design issues in combining veryhigh-speed silicon-germanium bipolar transistors and ULSI CMOS for system-on-a-chip applications," in Tech. Dig. IEDM, 1999, pp. 845-848.

[5] G. F. Niu, W. E. Ansley, S. Zhang, J. D. Cressler, C. S. Webster, and R. A. Groves, "Noise parameter optimization of UHV/CVD SiGe HBT's for RF and microwave applications," IEEE Trans. Electron Devices, vol. 46, pp. 1347-1354, Aug. 1999.

[6] G. Niu, J. D. Cressler, Z. Jin, S. Zhang, J. B. Juraver, M. Borgarino, R. Plana, and O. Llopis, "Transistor noise in SiGe HBT RF technology," in Proc. IEEE BCTM, 2000, pp. 207-210.

[7] D. B. Leeson, "A simple model of feedback oscillator noise spectrum," Proc. IEEE, vol. 54, pp. 329-330, Feb. 1966.

[8] P. Wambacq and W. Sansen, Distortion Analysis of Analog Integrated Circuits. Norwell, MA: Kluwer, 1998. 